Sādhanā Vol. 40, Part 3, May 2015, pp. 875-887. (C) Indian Academy of Sciences

\title{
Effect of coil embolization on blood flow through a saccular cerebral aneurysm
}

\author{
VISHAL AGRAWAL, CHANDAN PAUL, M K DAS* and \\ K MURALIDHAR
}

Department of Mechanical Engineering, Indian Institute of Technology Kanpur, Kanpur, UP 208016, India

e-mail: mkdas@iitk.ac.in

MS received 15 May 2014; revised 28 October 2014; accepted 20 November 2014

\begin{abstract}
Coil embolization is a mildly invasive endovascular method for treatment of a cerebral aneurysm. The presence of a coil reduces fluid loading of the blood vessel and delays further deformation of the walls. Its effectiveness depends on the coil porosity and permeability apart from the nature of flow pulsations and its geometry. In the present work, a three dimensional numerical study of pulsatile flow of blood through an artery with saccular cerebral aneurysm is reported. The flow is unsteady but is taken to be laminar and incompressible. The coil is treated as homogeneous and isotropic porous medium. A comparative study has been carried out on aneurysms with and without a coil insert considering blood as a non-Newtonian fluid. The simulation is carried out for Reynolds numbers $R e=500$ and 1500 . Results show that the velocity magnitude within the coil embolized aneurysm becomes negligible after coil insertion. The wall shear stress within the aneurysm decreases to a great extent for both Reynolds numbers. Pressure levels remain relatively unchanged. Overall, reduced wall loading with a coil stabilizes the growth of the aneurysm and thus provides an advantage.
\end{abstract}

Keywords. Coil embolization; hemodynamics; 3D-FVM; porous media; non-Newtonian fluid.

\section{Introduction}

Saccular cerebral aneurysm, also known as berry aneurysm refers to the cerebro-vascular disorder of weakening and outpouching of cerebral blood vessel (Yu \& Zhao 1999). Hemodynamic factors such as wall shear stress, wall pressure and the time-dependent blood flow rate play major roles in the initiation, evolution and the possible rupture of the aneurysm (Lieber et al 2002). Treatment options of cerebral aneurysm focus on reducing the wall pressure and shear

*For correspondence 
at the weakened blood vessel (Liou et al 2004). For instance, the coil embolization technique, a proposed procedure for aneurysm treatment attempts load reduction in the weakened vessel via insertion of a porous medium. The effectiveness of this procedure is judged by the extent to which it can alter wall loading in terms of pressure and shear stress. Over the years several experimental and numerical studies have been conducted for cerebral aneurysms. Canton et al (2005) experimentally studied the influence of stents on intra-aneurismal flow using an idealized geometry. Early studies on blood flow simulation through an aneurysm (Khanafer et al 2006) investigated pulsatile flow through a bulge (Sheard 2009) without any reference to the aneurysm treatment. Byun \& Rhee (2004) numerically studied coil embolization by modeling the coil as a small solid sphere placed at various locations within the aneurysm. Groden et al (2001) studied three dimensional pulsatile flow in a cerebral aneurysm numerically before and after coil embolization. The authors used cube-shaped cells to represent the coils. Preliminary studies have also been carried out treating coil embolization using porous medium models (Vafai 2011).

Transport through porous medium has been investigated experimentally, numerically and analytically by many researchers over the last few decades (Kaviany 1999). Earlier studies of porous medium dealt with the Darcy's law which relates flow velocity linearly to the pressure gradient. Later developments led to a refined model where two more forcing terms were added in the momentum equation. The Brinkman term captures the confining boundary effect while the Forchheimer term accounts for fluid acceleration (Nield \& Bejan 2006). While a few studies are available for fluid flow through a bulge, no specific study has been reported so far for pulsatile flow simulation through a bulge filled with porous medium using the Forchheimer-Brinkman extended Darcy model.

The present work develops an understanding of the effect of coil embolization on wall loading in terms of pressure and wall shear stress. The coil is treated as a homogeneous isotropic porous medium. Blood is treated as a non-Newtonian fluid. Distribution of the flow variables is captured within the aneurysm under pulsatile flow conditions and the effect of a coil in reducing wall loading is examined.

\section{Mathematical modeling}

The model geometry, governing equations, boundary conditions and the range of parameters are discussed in the present section.

\subsection{Geometry}

The geometry considered is a bent tube with a sphere-shaped aneurysm modeled as a bulge located at the central location (figure 1). The dimensions shown are greater by a factor of four in comparison to those of a human subject (Weir 1987). Other parameters are set in such a way that the dimensionless variables of the flow field, specifically Reynolds and Womersley numbers ( $\mathrm{Re}$ and Wo) are matched (Section 2.3). The angle between the inlet and outlet of the tube is kept as $75^{\circ}$. For the model in figure 1 , the bulge parameter is $D / d=3$. The lengths of the inlet and exit sections are longer than in a human patient and are selected to minimize flow development effects. Real aneurysms of the shape considered in figure 1 have been found in patients (Gobin et al 1994). In addition, it is known that aneurysms are prone to appear at tube junctions, bends, and bifurcations (Sazonov et al 2011). 


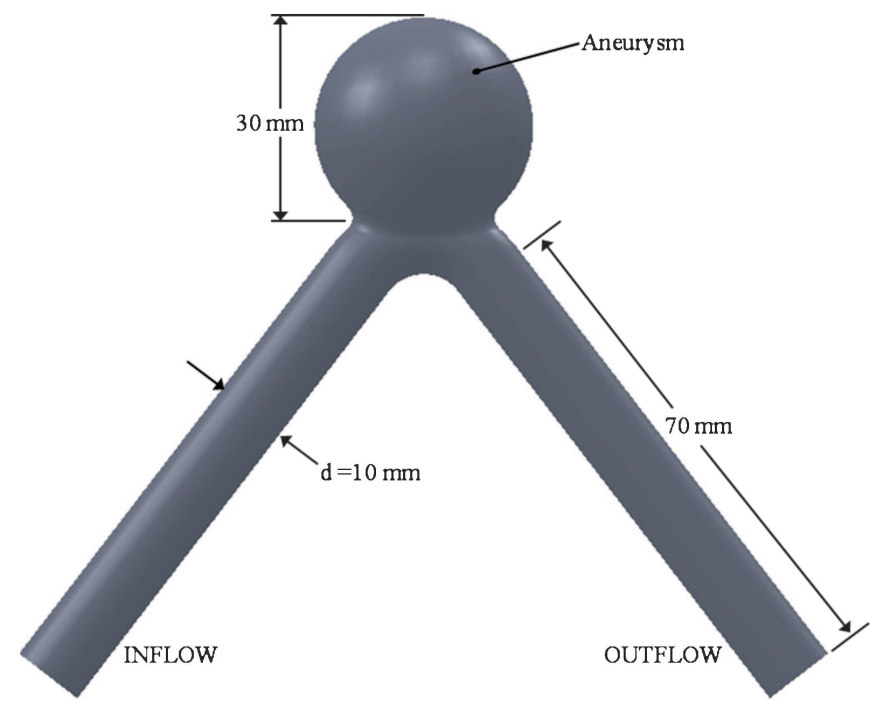

Figure 1. Schematic diagram of the geometric model considered for analysis.

\subsection{Governing equations and boundary conditions}

Pulsatile flow of blood through the bulge is unsteady but is considered incompressible and laminar. The porous medium fills the bulge but the legs carry clear fluid. The dimensionless form of the governing equations in index notation for incompressible fluid flow through clear and porous media is given below (Nield \& Bejan 2006):

$$
\begin{gathered}
\frac{\partial u_{i}}{\partial x_{i}}=0 \\
\frac{\partial u_{i}}{\partial t}+u_{j} \frac{\partial u_{i}}{\partial x_{j}}=-\frac{\partial p}{\partial x_{i}}+\frac{1}{\operatorname{Re}} \frac{\partial}{\partial x_{j}}\left(\frac{\mu}{\mu_{\infty}}\left[\frac{\partial u_{i}}{\partial x_{j}}+\frac{\partial u_{j}}{\partial x_{i}}\right]\right)-\frac{\phi}{\operatorname{ReDa}} \frac{\mu_{\infty}}{\mu} u_{i}-\frac{C_{f} \phi}{\sqrt{\mathrm{Da}}}|\mathbf{u}| u_{i}
\end{gathered}
$$

The meaning of each symbol is given in the nomenclature. For a porous medium, symbol $u_{i}$ represents the volumetric phase-averaged velocity and $p$ is intrinsic phase-averaged pressure. The last three expressions on the right side of Eq. (2) are referred as the Brinkman, Darcy, and the Forchheimer terms. Darcy and Forchheimer terms are set to zero in the clear medium. The terms used for nondimensionalizing the governing equations are listed in table 1. For a

Table 1. Terms used for nondimensionalization.

\begin{tabular}{llll}
\hline Length $(\mathrm{m})$ & $d$ & Velocity $(\mathrm{m} / \mathrm{s})$ & $U_{\text {ref }}$ \\
Pressure $(\mathrm{Pa})$ & $\rho\left(U_{\text {ref }}\right)^{2}$ & Vorticity $\left(\mathrm{s}^{-1}\right)$ & $U_{\text {ref }} / d$ \\
WSS $(\mathrm{Pa})$ & $\mu_{\infty} U_{\text {ref }} / d$ & Time $(\mathrm{s})$ & $d / U_{\text {ref }}$ \\
Angular frequency $(\mathrm{Hz})$ & $U_{\text {ref }} / d$ & Viscosity (Pa-s) & $\mu_{\infty}$ \\
\hline
\end{tabular}


Newtonian fluid, $\mu / \mu_{\infty}$ is unity. The shear-thinning characteristics of blood are incorporated using the Carreau-Yasuda Model (Leuprecht \& Perktold 2001):

$$
\mu(\dot{\gamma})=\mu_{\infty}+\frac{\mu_{0}-\mu_{\infty}}{\left(1+m \dot{\gamma}^{n}\right)^{a}} \text { where } \dot{\gamma}=\left(\frac{1}{2} e_{i j} e_{i j}\right)^{\frac{1}{2}} \text { and } e_{i j}=\frac{1}{2}\left(\frac{\partial u_{i}}{\partial x_{j}}+\frac{\partial u_{j}}{\partial x_{i}}\right)
$$

Here $\mu_{o}$ and $\mu_{\infty}$ represent the viscosity at zero and infinite shear rate respectively and $m$ denotes the relaxation time. The shear rate $(\dot{\gamma})$ is obtained from the strain-rate tensor $\left(e_{i j}\right)$ as defined above.

At the inflow plane, the Womersley profile (Womersley 1955) derived from a physiological velocity waveform of internal carotid artery is specified as the boundary condition for both clear and porous media (Valencia et al 2008). The no slip condition is used at the solid walls. Pressure is specified as zero (datum) at the outlet while for velocity, the gradient outflow condition is prescribed. The time-dependent waveform for the inlet velocity profile is shown in figure 2 .

\subsection{Flow parameters}

The parameters of the Carreau-Yashuda model for blood viscosity are (Eq. 3): $\mu_{o}=0.16$ Pa-s, $\mu_{\infty}=0.0035 \mathrm{~Pa}-\mathrm{s}, m=8.2, n=0.64$ and $a=1.23$. In addition, density $\rho=1050 \mathrm{~kg} / \mathrm{m}^{3}$. The parameters for porous media are: porosity $\varphi=0.735$, permeability $K=1.55 \times 10^{-8} \mathrm{~m}^{2}$ and the inertia coefficient $C_{f}$ is 0.2 (Kakalis et al 2008). The Darcy number (Da) of the coil can now be calculated as $K / D^{2}=1.55 \times 10^{-4}$. Once the artery diameter, density and viscosity are fixed, the reference velocity $U_{\text {ref }}$ is obtained from the set Reynolds number $(\mathrm{Re})$. The values of $U_{\text {ref }}$ are found to be $0.167 \mathrm{~m} / \mathrm{s}$ and $0.5 \mathrm{~m} / \mathrm{s}$ for $R e=500$ and 1300 respectively. For a $R e$ of 500 and a pulsing frequency of $f=1.17 \mathrm{~Hz}$, the Womersley number $\left(\mathrm{Wo}_{0}=\frac{d}{2} \sqrt{\frac{2 \pi f \rho}{\mu_{\infty}}}\right)$ is 7.8. This

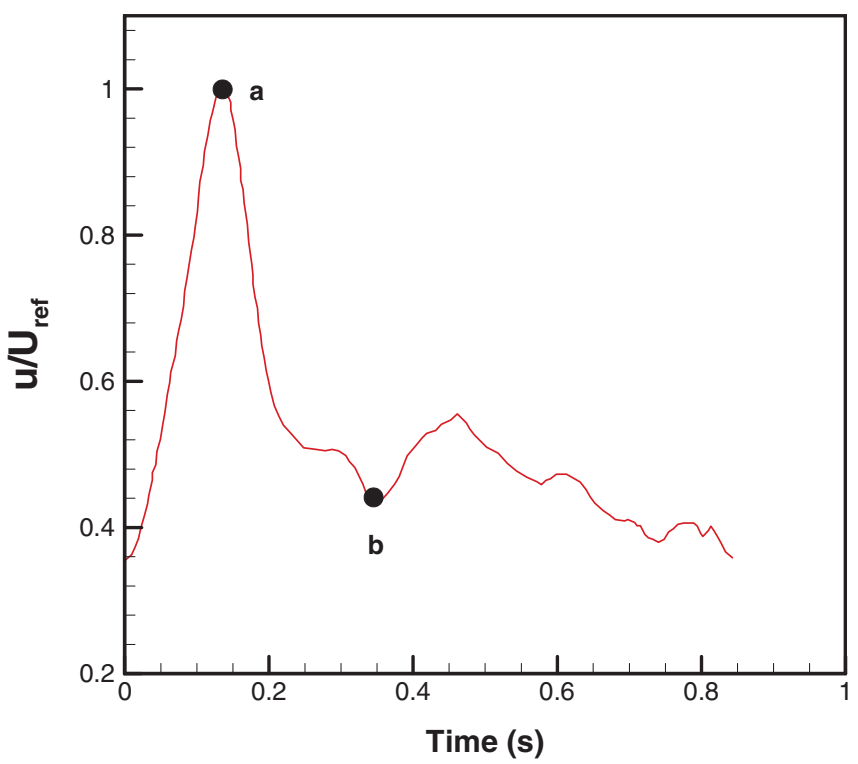

Figure 2. Inlet average velocity waveform (Valencia et al 2008). Points a-b indicate the time instants at which the solution has been presented in Section 4. 
values of Re and Wo are consistent with oscillatory flow occurring in the cerebral region of the human body.

\section{Numerical simulation}

The unsteady governing equations of flow in clear and porous media are solved using the finite volume method (FVM) on 3D unstructured tetrahedral meshes. ANSYS-ICEM tool is used to generate high quality unstructured tetrahedral meshes within the geometry. A method of solution for the transport equations on unstructured meshes with cell-centered collocated variables approach was discussed earlier by Gohil et al (2012). This approach has been extended to incorporate Darcy and Forchheimer terms in the governing equation as well as the non-Newtonian nature of blood. The mesh used for the simulation is quite refined with over $1.1 \times 10^{6}$ elements. It contains a total of 1,92,640 nodes to form a tetrahedral mesh where each cell is highly regular with a near-unity aspect ratio. The grid quality of the mesh was ascertained to be greater than 0.9 at all locations. Code validation of the FVM solver is further discussed by Sikarwar et al (2013).

\section{Results and discussion}

Quantities of interest are flow distribution in the bulge along with the wall shear stress and wall pressure. Data have been presented for the region close to the bulge since the legs are less

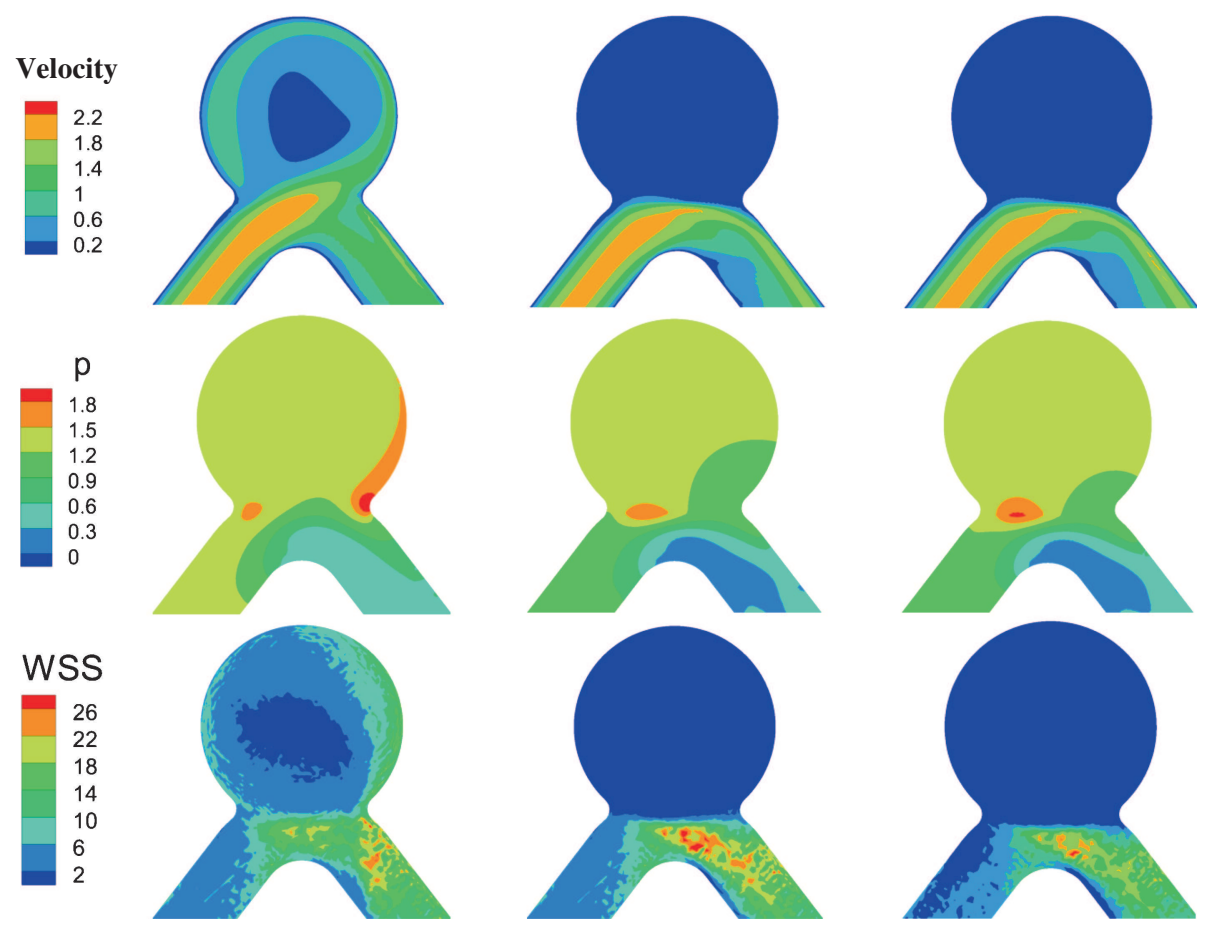

Figure 3. Time-averaged distribution of absolute velocity (at mid-plane in front view), wall pressure and wall shear stress for clear medium (first column), porous medium (second column) and porous medium with non-Newtonian flow (third column) at Reynolds number $R e=500$ and Womersley number $W o=7.82$. 
important for the present study. The time-averaged flow in the model is small but non-zero and net flow prevails form left to right. However, the oscillatory part of the flow is quite significant and flow distribution has been shown in terms of the RMS values. From a biological perspective, static loading is not as critical as the time-dependent counterpart since the latter, by the mechanism of fatigue, leads to progressive failure of the walls of the blood vessel. In the following paragraphs, Re of 500 and 1500 are compared at a Wo of 7.82. Flow in a clear geometry, one with a coil in the bulge, and simulation of blood as a non-Newtonian fluid are compared. Timeaveraged and RMS values are obtained by averaging the flow signals at each cell over three cycles of figure 2. The differences between Newtonian and non-Newtonian characteristics are seen in the present study to be small. However, the presence of a coil substantially alters the flow properties in the bulge.

Figure 3 shows the time-averaged distribution of total velocity, wall pressure and wall shear stress around the bulge. The first column is for a clear bulge, the second is for the bulge with a coil, and the third column presents data when the fluid is taken to be non-Newtonian. For a clear bulge, a certain fluid velocity is attained within, though the flow shows circulation. With a coil in place, the main stream bypasses the bulge, and velocities obtained within are much smaller - by a factor of ten, essentially showing a suppression of flow in this region. The slow flow through the bulge with a coil is unidirectional and does not classify as flow separation. The
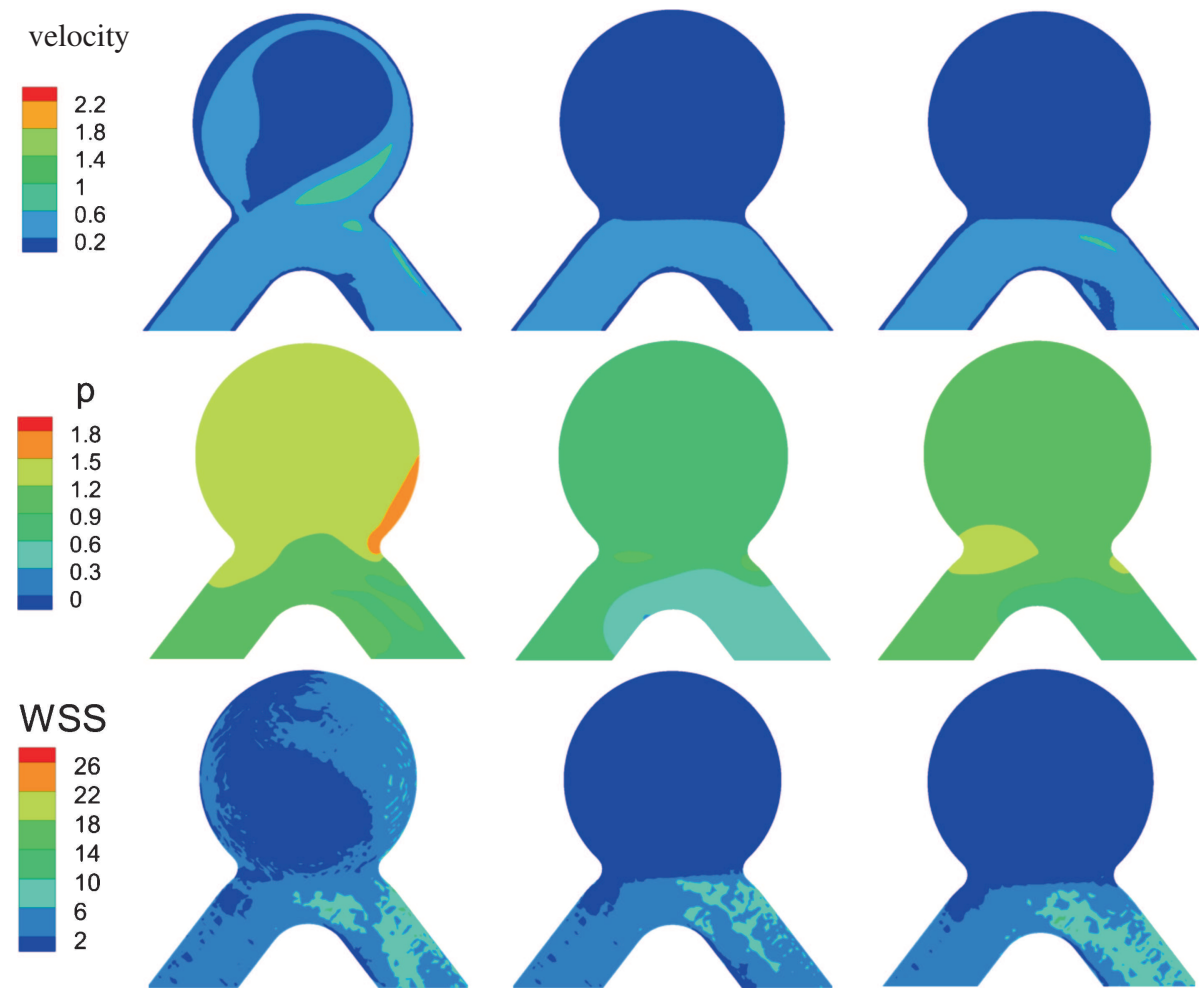

Figure 4. RMS deviation of absolute velocity (at mid-plane in front view), wall pressure and wall shear stress from the time-averaged values of the flow variables for clear medium (first column), porous medium (second column) and porous medium with non-Newtonian flow (third column) at Reynolds number $R e=500$ and Womersley number $W o=7.82$. 
results obtained with a non-Newtonian fluid model are quite similar. A reduction in flow in the bulge shows a simultaneous reduction in wall shear stress - by a factor of 8 . For pressure, the bulge is a zone of separation and hence spatially uniform pressure. This trend continues with the bulge carrying the coil, uniform pressure arising from slow unidirectional flow through the model. Figure 3 also shows that the increase in overall resistance to flow in the presence of a coil is small, mainly because flow has bypassed the bulge region.

Figure 4 presents RMS variation of total velocity, wall pressure, and wall shear stress after removing the time-averaged value at every cell. The trends here are similar to figure 3 in the sense that (a) flow in the bulge significantly reduces in the presence of a coil, (b) coil reduces the wall shear stress, and (c) the difference between Newtonian and non-Newtonian treatments is small. The changes in RMS wall pressure are however quite small. Consequently, wall pressure is mostly unaffected by the presence of the coil in the bulge. This result arises from the small absolute velocity prevailing in the bulge with and without the coils in place.

Calculations were repeated at a Re of 1500 for the geometry of interest (see figure 5 for RMS data). The trends seen at $R e=500$ were realized here as well, except that the velocity magnitude in the bulge was higher; so was the dimensionless pressure magnitude. With the insertion of a coil, velocity and shear stress values fell by nearly a factor of ten. The pressure values stayed
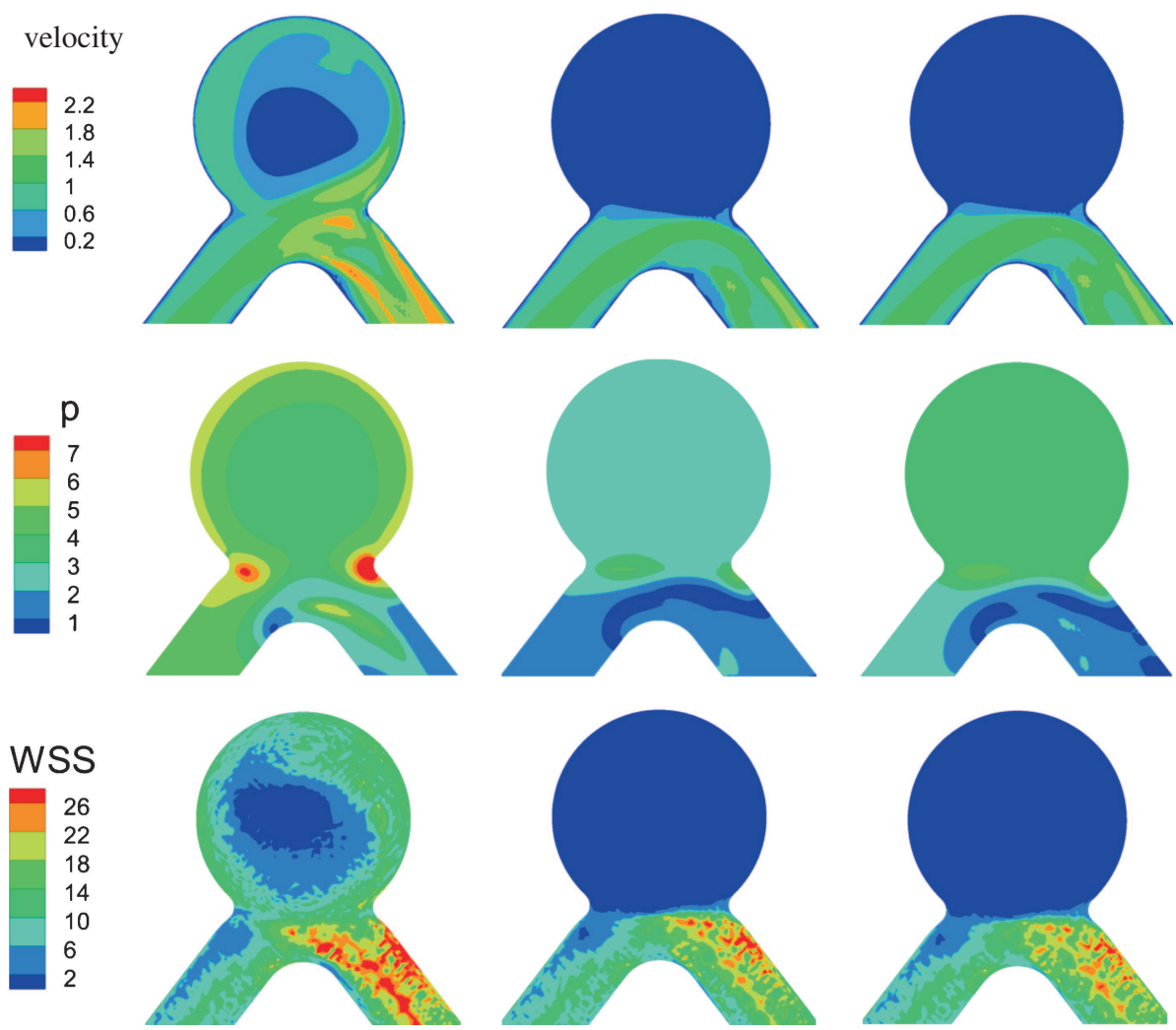

Figure 5. RMS deviation of absolute velocity (at mid-plane in front view), wall pressure and wall shear stress from the time-averaged values of the flow variables for clear medium (first column), porous medium (second column) and porous medium with non-Newtonian flow (third column) at Reynolds number $R e=1500$ and Womersley number $W o=7.82$. 
nearly constant. Hence, the wall loading of a clear bulge increased with Re but diminished in the presence of the coil. This trend is significant because it proves the utility of using a coil to stabilize wall loading over a range of Re.

Figures 6 and 7 present instantaneous flow data at phases ' $a$ ' and ' $b$ ' defined in figure 2. These correspond to the peak phase and one of the prominent minima. The flow direction is from left to right in both instances, except for a difference in magnitude. Reynolds numbers of 500 and 1500 are jointly compared. Figure 6 is for clear medium while figure 7 includes a coil placed in the bulge, the fluid being non-Newtonian.

The bulge experiences flow separation at the two time instants studied and at both Reynolds numbers. At phase 'a', flow in the bulge is stronger than at ' $b$ ' and the corresponding velocities, shear, and pressure are greater as well for a clear medium. With the placement of a coil, circulating velocities in the bulge reduce, wall shear reduces, while pressure levels remain relatively unaffected. Instantaneous flow patterns confirm that the coil creates a bypass that stabilizes wall loading of the bulge. Thus, the instantaneous variation in the flow properties from phase ' $a$ ' to ' $b$ ' is analogous to a change in Reynolds number in the time-averaged and RMS data.

Apart from overall trends in the bulge, it is of interest to know if the loading is particularly high at selected locations. For this purpose, instantaneous wall shear and pressure are provided

Phase a

$$
\operatorname{Re}=500
$$

Phase b
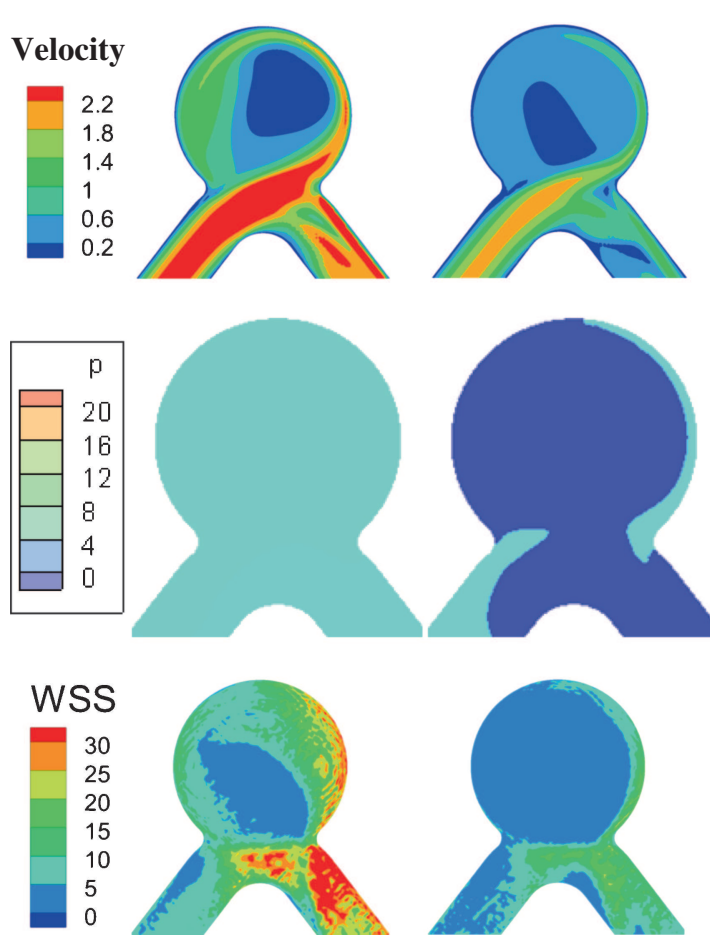

$R e=1500$

Phase b

Phase a
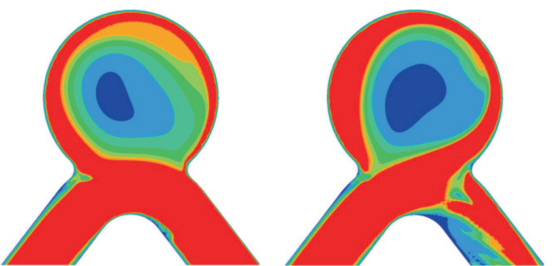

Figure 6. Comparison of absolute velocity (at mid-plane in front view), wall pressure and wall shear stress in clear media at selected phases of the cycle as shown in figure 2 for Reynolds number, $R e=500$ and $R e=1500$ and Womersley number, $W o=7.82$. 
Phase a

$$
R e=500
$$
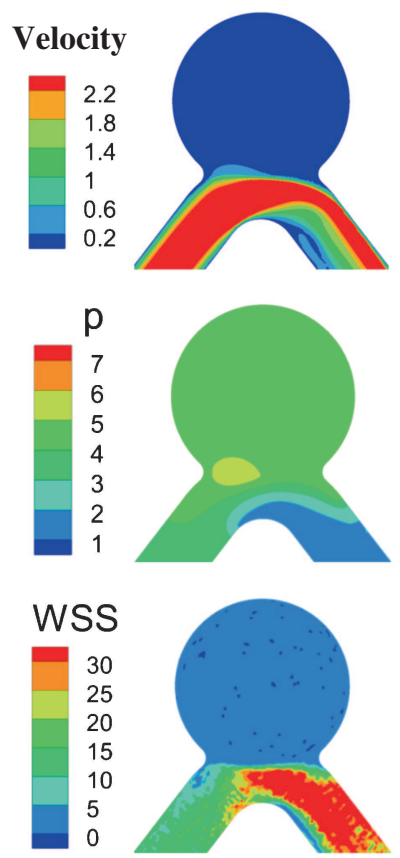

Phase b
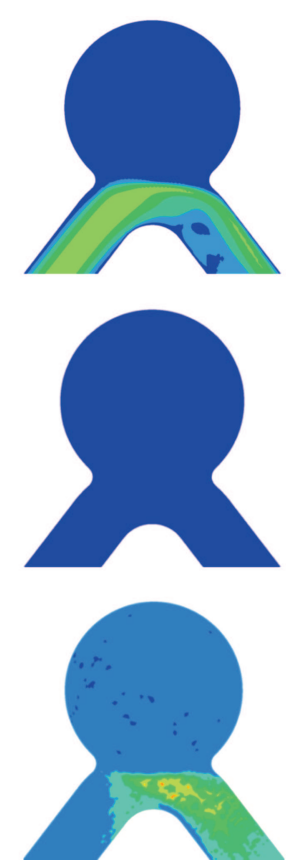

Phase a

Phase b

$R e=1500$

Figure 7. Comparison of absolute velocity (at mid-plane in front view), wall pressure and wall shear stress in porous media with non-Newtonian model at selected phases of the cycle as shown in figure 2 for Reynolds number, $R e=500$ and $R e=1500$ and Womersley number, $W o=7.82$.

in figure 8 at points $\mathrm{A}, \mathrm{B}$, and $\mathrm{C}$ of the bulge. Reynolds numbers of 500 and 1500 are shown. Wall shear stresses at points A, B, and C are distinctly different for a bulge with clear fluid. In the presence of a coil, the flow pattern is regularized and the differences are smaller. Wall shear stresses increase substantially with Reynolds number in a clear bulge but the increase is smaller in the bulge with a coil. For a clear bulge, point A shows the largest wall shear and is expected to be the point of highest wall loading. Point B shows lower wall shear over a cycle and is also a point of interest. These differences are broadly submerged when a coil is used since the flow in the bulge is homogenized.

The pressure variation, plotted in figure 8 , shows that the three points A, B and C are largely indistinguishable in terms of pressure and consistently reveal the effect of Re. Thus, pressure is spatially uniform in the bulge at all time instants. Pressure increases with Re and is slightly lowered when the coil is placed within the aneurysm. A non-Newtonian fluid treatment shows a slight augmentation in pressure because of the shear-thinning behavior of the fluid. As shown in figure 8 , the presence of a porous medium inside the bulge softens the flow pulsation and prohibits the inflow pulsation to reach the wall. A comparison between figure 8(ii) and (iii) shows that Newtonian fluid suppresses flow pulsation more efficiently than the shear-thinning fluid. Suppression of flow pulsation indicates the dominance of diffusive momentum-transport promoted by the presence of the porous medium. The shear thinning rheology of blood, on the other hand, reduces viscosity in the regions of high velocity gradients promoting the convective and 


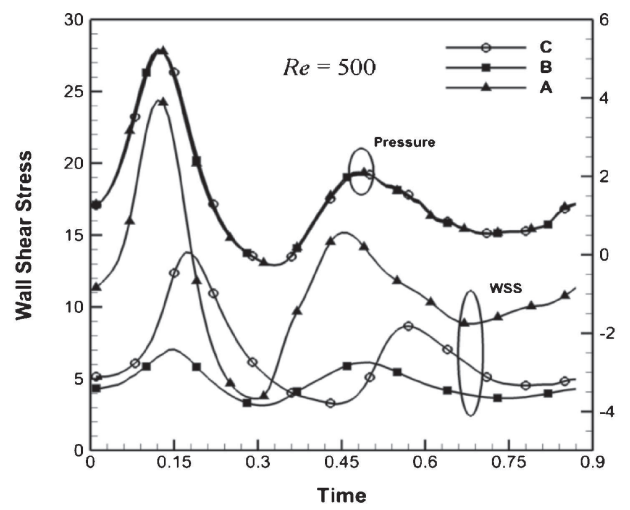

(i)

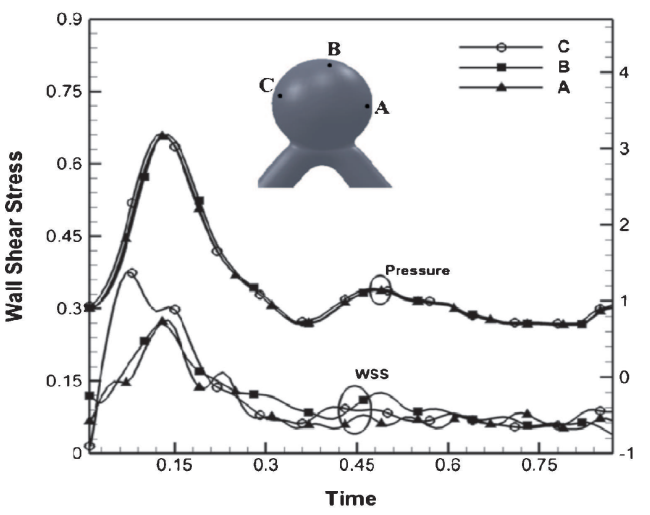

(ii)

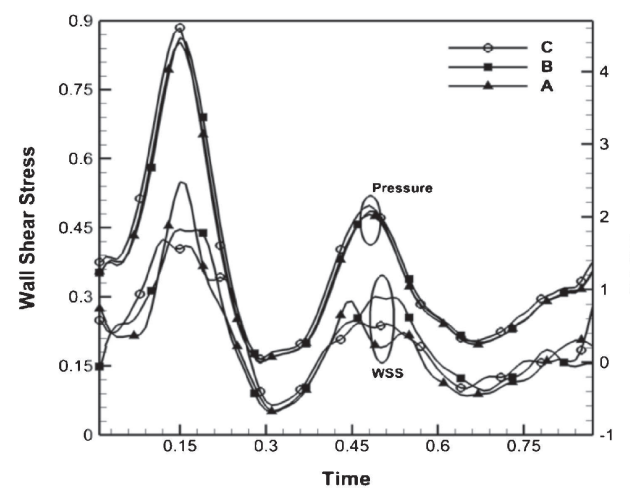

(iii)

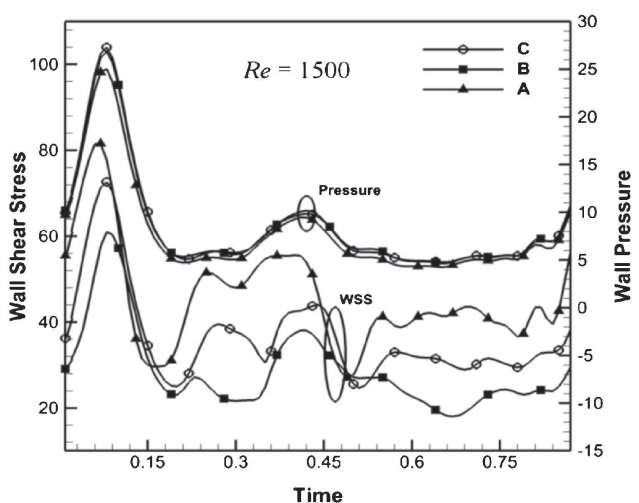

(i)

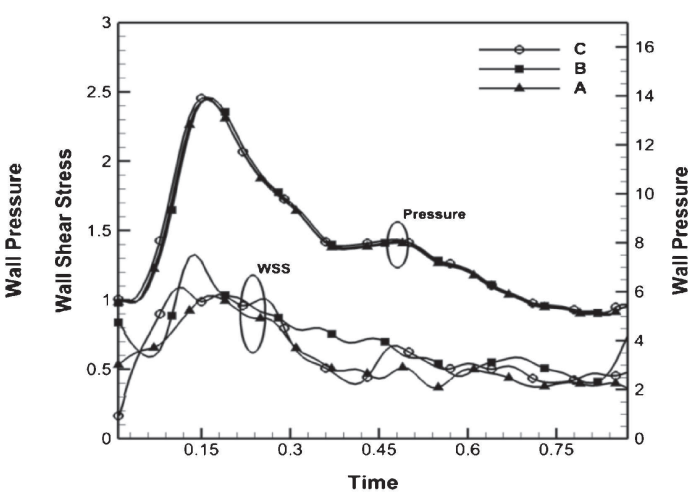

(ii)

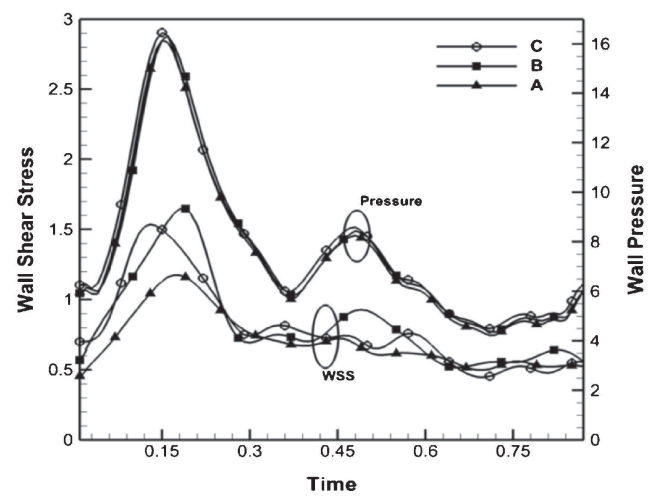

(iii)

Figure 8. Wall shear stress and pressure variation with time at selected points $\mathrm{A}, \mathrm{B}$ and $\mathrm{C}$ in the bulge region for (i) clear media, (ii) porous media and (iii) porous media with non-Newtonian flow at $R e=500$ (left column) and $R e=1500$ (right column).

Darcian transport mechanisms. Dominance of such transport mechanisms preserves the inflow pulsation, as shown in figure 8(iii). The shear thinning behavior of blood dominates the near-wall region of the blood vessel, including points $\mathrm{A}, \mathrm{B}$, and $\mathrm{C}$. These points, therefore, experience the 
rheology-induced low viscosity effect and transport the inflow pulsation up to the wall, as shown in figure 8(iii).

\subsection{Solid particulates of blood}

Blood is a multi-phase fluid mixture that includes particulate matter. The solid phase contains red blood cells (RBCs), white blood cells (WBCs) and platelets. These elements are carried in the liquid phase, called the plasma containing organic molecules, such as enzymes, antibodies, and electrolytes. Several studies have been conducted over the years to study the nature of solid particles in blood flow. Boryczko et al (2003) modeled the dynamics of red blood cells and plasma using a discrete particle approach. It is now understood that the solid phase constitutes almost $45 \%$ of the total blood composition - red blood cells are predominant. A measure of solid particles is given by the blood hematocrit which represents the volume fraction of RBCs present in the blood. For an average person, the hematocrit is about $45 \%$. Platelets occupy $2 \%$ while WBCs are about $0.5 \%$ of the volume. The sizes of the blood cells in the solid phase vary from $5 \mu \mathrm{m}$ to $20 \mu \mathrm{m}$ in case of RBC to WBC, respectively. The dynamic clustering of red blood cells leads up to a dimension of 20-30 $\mu \mathrm{m}$. The aneurysm region in the model used is quite large, with a diameter of $30 \mathrm{~mm}$. The packing density by volume for the coils placed will be $26.5 \%$ (porosity 0.735 ). The pore length scale for this volume can now be estimated as $10 \mathrm{~mm}$. Since the pore-scale is much larger than the blood particles, one can make an approximation that the coil will not selectively block the passage of particulates. The possibility of the coil porosity diminishing with time is also minimal.

\section{Conclusions}

In the present study coil embolization modeling of saccular cerebral aneurysm is reported. The coils are modeled as porous medium and represented using Forchheimer-Brinkman extended Darcy model. A realistic velocity waveform is applied on the inflow plane. The unsteady form of the governing equations is solved in three dimensions by a finite volume method. Results obtained in the present work show that the bulge is a region of flow recirculation. In the presence of a coil, the bulge is bypassed and flow intensity in the bulge is greatly reduced. Consequently, wall shear stress is also diminished. The pressure distribution in the bulge remains spatially uniform with and without the coil. The effect of treating blood as non-Newtonian is to increase wall shear and pressure levels, though the differences with respect to the Newtonian model are only marginal. The particulate nature of blood is not expected to alter the results obtained since the suspended RBCs are much smaller than the pore dimensions of the coil.

\section{Nomenclature}

$\begin{array}{ll}C_{f} & \text { inertia coefficient } \\ d & \text { diameter of the tube }(\mathrm{m}) \\ D & \text { diameter of the bulge }(\mathrm{m}) \\ \mathrm{Da} & \text { Darcy Number } \\ f & \text { frequency }(\mathrm{Hz}) \\ K & \text { permeability }\left(\mathrm{m}^{2}\right)\end{array}$




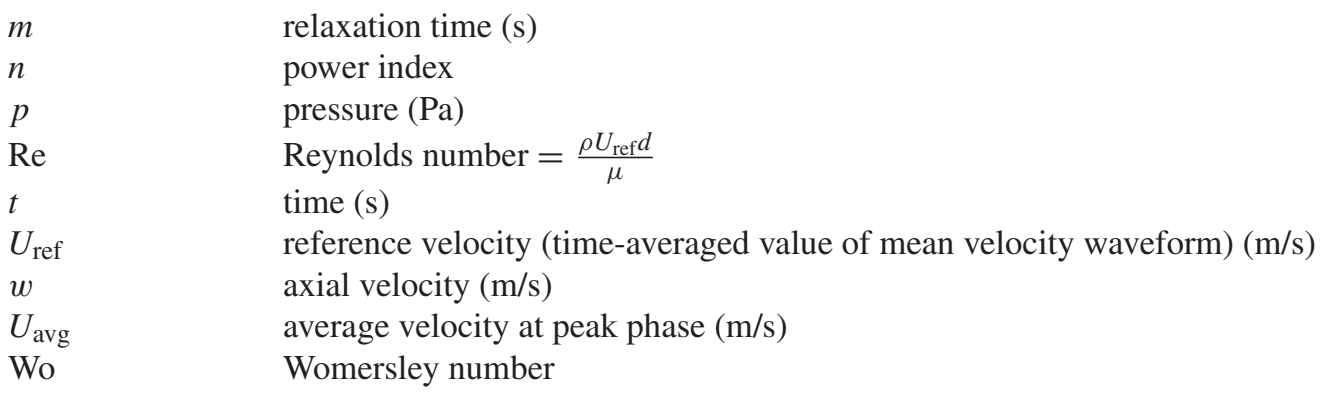

Greek symbols

$\begin{array}{ll}\rho & \text { density }\left(\mathrm{kg} / \mathrm{m}^{3}\right) \\ \varphi & \text { porosity } \\ \mu & \text { dynamic viscosity }(\mathrm{Pa}-\mathrm{s})\end{array}$

\section{References}

Boryczko K, Dzwinel W and Yuen D A 2003 Dynamical Clustering of Red Blood Cells in Capillary Vessels. J. Mol. Mod. 1: 16-33

Byun H S and Rhee K 2004 CFD modeling of blood flow following coil embolization of aneurysms. Med. Eng. Phys. 26: 755-761

Canton G, Levy D I and Lasheras J C 2005 Changes in the intra-aneurysmal pressure due to hydrocoil embolization. AJNR Am. J. Neuroradiol. 26: 904-907

Gobin Y P, Counord J L, Flaud P and Duffaux J 1994 In vitro study of haemodynamics in a giant saccular aneurysm model: influence of flow dynamics in the parent vessel and effects of coil embolisation. Neuroradiology 36(7): 530-536

Groden C, Laudan J, Gatchell S and Zeumer H 2001 Three-dimensional pulsatile flow simulation before and after endovascular coil embolization of a terminal cerebral aneurysm. J. Cerebral Blood Flow Metab. 21: 1464-1471

Gohil T, McGregor T R, Szczerba D, Burckhardt K, Muralidhar K and Szekely G 2012 Simulation of oscillatory flow in an aortic bifurcation using FVM and FEM: a comparative study. Int. J. Num. Methods Fluid. 66: 1037-1067

Kakalis N M P, Mitsos A P, Byrne J V and Ventikos Y 2008 The haemodynamics of endovascular aneurysm treatment: a computational modelling approach for estimating the influence of multiple coil deployment. IEEE Trans. Med. Imaging 27(6): 814-824. doi:10.1109/TMI.2008.915549

Kaviany M 1999 Principles of heat transfer in porous media. New York: Springer

Khanafer K M, Gadhoke P, Berguer R and Joseph L B 2006 Modeling pulsatile flow in aortic aneurysms: Effect of non-Newtonian properties of blood. Biorheology, IOS Press 43: 661-679

Leuprecht A and Perktold K 2001 Computer simulation of non-Newtonian effects of blood flow in large arteries. Comput. Methods Biomech. Eng. 4: 149-163

Lieber R B, Livescu V, Hopkins L N and Wakhiloo A K 2002 Particle image velocimetry assesment of stent design influence on intra-aneurysmal flow. Annals Biomed. Eng. 30: 768-777

Liou T M, Liou S M and Chu K L 2004 Intra-aneurysmal flow with helix and mesh stent placement across side-wall aneurysm pore of a straight parent vessel. ASME J. Biomech. Eng. 126: 36-43

Nield D A and Bejan A 2006 Convection in porous media, Third edition ed. New York: Springer-Verlag

Sazonov I, Yeo S Y, Bevan R L T, Xie X, Loon R van and Nithiarasu P 2011 Modeling Pipeline for Subject-specific Blood Flow - A Review. Int. J. Num. Methods Biomed. Eng. 27: 1868-1910 
Sheard G J 2009 Flow dynamics and wall shear-stress variation in a fusiform aneurism. J. Eng. Math. 64: 379-390

Sikarwar B S, Khandekar S and Muralidhar K 2013 Mathematical modeling of dropwise condensation on textured surfaces. Sadhana 38: 1-37

Vafai K 2011 Porous media: applications in biological systems and biotechnology. Boca Raton, FL: CRC Press, Taylor and Frnacis Group, 33487: 2742

Valencia A, Morales H, Rivera R, Bravo E and Galvez M 2008 Blood flow dynamics in patient-specific cerebral aneurysm models: The relationship between wall shear stress and aneurysm area index. Med. Eng. Phys. 30(3): 329-340. doi:10.1016/j.medengphy.2007.04.011

Weir B 1987 Aneurysms affecting the nervous system: Williams and Wilkins

Womersley J R 1955 Velocity profiles of oscillating arterial flow, with some calculation of the viscous drag and the Reynolds Number. J. Physiol. 128: 629-640

Yu S C M and Zhao J B 1999 A steady flow analysis on the stented and non-syented sidewall aneurysm models. Med. Eng. Phys. 21: 133-141 